OPEN ACCESS

Edited by:

Thomas Tessonnier,

Heidelberg University Hospital,

Germany

Reviewed by:

Stefan Koerber,

Heidelberg University Hospital,

Germany

Hirokazu Makishima

University of Tsukuba, Japan

*Correspondence:

Stefania Russo

stefania.russo@cnao.it

Rosalinda Ricotti

rosalinda.ricotti@cnao.it

Barbara Vischion

barbara.vischioni@cnao.it

${ }^{+}$These authors have contributed

equally to this work and

share first authorship

tThese authors have contributed

equally to this work and

share last authorship

Specialty section:

This article was submitted to Radiation Oncology,

a section of the journal

Frontiers in Oncology

Received: 13 July 2021 Accepted: 06 September 2021 Published: 28 September 2021

Citation:

Russo S, Ricotti R, Molinelli S, Patti F, Barcellini A, Mastella E, Pella A,

Paganelli C, Marvaso G, Pepa M,

Comi S, Zaffaroni M, Avuzzi B,

Giandini T, Pignoli E, Valdagni $R$, Baroni G, Cattani F, Ciocca M,

Jereczek-Fossa BA, Orlandi E, Orecchia $R$ and Vischioni B (2021) Dosimetric Impact of Inter-Fraction Anatomical Changes in Carbon Ion Boost Treatment for High-Risk Prostate Cancer (AIRC IG 14300).

Front. Oncol. 11:740661. doi: 10.3389/fonc.2021.740661

\section{Dosimetric Impact of Inter-Fraction Anatomical Changes in Carbon Ion Boost Treatment for High-Risk Prostate Cancer (AIRC IG 14300)}

\author{
Stefania Russo ${ }^{1 * t}$, Rosalinda Ricotti ${ }^{2 * t}$, Silvia Molinelli ${ }^{1}$, Filippo Patti ${ }^{3,4}$, \\ Amelia Barcellini ${ }^{3}$, Edoardo Mastella ${ }^{1}$, Andrea Pella ${ }^{2}$, Chiara Paganelli ${ }^{5}$, \\ Giulia Marvaso ${ }^{4,6}$, Matteo Pepa ${ }^{4}$, Stefania Comi ${ }^{7}$, Mattia Zaffaroni ${ }^{4}$, Barbara Avuzzi ${ }^{8}$, \\ Tommaso Giandini ${ }^{9}$, Emanuele Pignoli ${ }^{9}$, Riccardo Valdagni ${ }^{6,7}$, Guido Baroni ${ }^{2,5}$, \\ Federica Cattani ${ }^{7}$, Mario Ciocca ${ }^{1}$, Barbara Alicja Jereczek-Fossa ${ }^{4,6}$, Ester Orlandi ${ }^{3}$, \\ Roberto Orecchia ${ }^{10 \neq}$ and Barbara Vischioni ${ }^{{ }^{* \neq}}$ \\ ${ }^{1}$ Medical Physics Unit, Clinical Department, National Center for Oncological Hadrontherapy (CNAO), Pavia, Italy, \\ ${ }^{2}$ Bioengineering Unit, Clinical Department, National Center for Oncological Hadrontherapy (CNAO), Pavia, Italy, \\ ${ }^{3}$ Radiotherapy Unit, Clinical Department, National Center for Oncological Hadrontherapy (CNAO), Pavia, Italy, ${ }^{4}$ Division of \\ Radiotherapy, IEO, European Institute of Oncology Istituto di Ricovero e Cura a Carattere Scientifico (IRCCS), Milan, Italy, \\ ${ }^{5}$ Department of Electronics, Information and Bioengineering, Politecnico di Milano, Milan, Italy, ${ }^{6}$ Department of Oncology \\ and Hemato-oncology, University of Milan, Milan, Italy, ${ }^{7}$ Medical Physics Unit, IEO, European Institute of Oncology Istituto di \\ Ricovero e Cura a Carattere Scientifico (IRCCS), Milan, Italy, ${ }^{8}$ Department of Radiation Oncology, Fondazione Istituto di \\ Ricovero e Cura a Carattere Scientifico (IRCCS) Istituto Nazionale dei Tumori, Milan, Italy, ${ }^{9}$ Medical Physics Unit, Fondazione \\ Istituto di Ricovero e Cura a Carattere Scientifico (IRCCS) Istituto Nazionale dei Tumori, Milan, Italy, ${ }^{10}$ Scientific Directorate, \\ IEO, European Institute of Oncology Istituto di Ricovero e Cura a Carattere Scientifico (IRCCS), Milan, Italy
}

Rectum and bladder volumes play an important role in the dose distribution reproducibility in prostate cancer adenocarcinoma ( $\mathrm{PCa}$ ) radiotherapy, especially for particle therapy, where density variation can strongly affect the dose distribution. We investigated the reliability and reproducibility of our image-guided radiotherapy (IGRT) and treatment planning protocol for carbon ion radiotherapy (CIRT) within the phase II mixed beam study (AIRC IG 14300) for the treatment of high-risk PCa. In order to calculate the daily dose distribution, a set of synthetic computed tomography (SCT) images was generated from the cone beam computed tomography (CBCT) images acquired in each treatment session. Planning target volume (PTV) together with rectum and bladder volume variation was evaluated with sCT dose-volume histogram (DVH) metric deviations from the planning values. The correlations between the bladder and rectum volumes, and the corresponding DVH metrics, were also assessed. No significant difference in the bladder, rectum, and PTV median volumes between the planning computed tomography ( $\mathrm{PCT}$ ) and the $\mathrm{sCT}$ was found. In addition, no significant difference was assessed when comparing the average DVHs and median DVH metrics between PCT and SCT. Dose deviations determined by bladder and rectum filling variations demonstrated that dose distributions were reproducible in terms of both target coverage and organs at risk (OARs) sparing.

Keywords: carbon ion radiotherapy (CIRT), high-risk prostate cancer, image-guided radiotherapy (IGRT), inter-fraction anatomical changes, dose-of-the-day calculation, deformable image registration (DIR) 


\section{INTRODUCTION}

Essential issues in prostate cancer adenocarcinoma $(\mathrm{PCa})$ irradiation are prostate motion and shape variations due to rectum and/or bladder filling modifications (1), which may strongly affect the target dose distribution and Organs at Risk (OAR) sparing (2). In order to maintain consistent rectum and bladder volume throughout the treatment, preparation instructions about food and fluid intake are usually given to each patient before treatment simulation and delivery. Despite this, inter-fractional unpredictable OAR volume variation might occur, and the reproducibility of dose distribution remains essential to providing an adequate and safe treatment of patients.

In this context, image-guided radiation therapy (IGRT) is essential to ensuring treatment efficacy and safety. In recent years, the introduction of new advanced techniques of IGRT using online cone beam computed tomography (CBCT) allows the tracking of daily positioning and anatomical changes of patients in treatment position. It also has the potential to be used to evaluate the dose-of-the-day distributions in comparison to the dose distribution calculated on the planning computed tomography (pCT) (3).

Since 2016 at the Centro Nazionale di Adroterapia Oncologica (CNAO, Pavia, Italy), we have enrolled patients in the phase II clinical trial with a mixed-beam approach for prostate irradiation, in collaboration with Istituto Europeo di Oncologia IRCCS (IEO) and Fondazione IRCCS Istituto Nazionale dei Tumori (INT) in Milan, Italy. The irradiation scheme consisted of a hypo-fractionated carbon ion radiotherapy (CIRT) anticipated boost to the prostate, followed by photon intensity modulated radiation therapy (IMRT) to the prostate and pelvic lymph nodes (grant AIRC IG 14300) (4).

The rationale of the hypo-fractionated CIRT boost is to escalate the biological dose to the target by exploiting carbon ion favorable physical and biological properties. The higher radiobiological effectiveness (RBE) of carbon ions on cancer radioresistant clones and more hypoxic tumor components (5) should enhance the efficacy of the subsequent photon phase of the scheme, delivered with conventional fractionation. Safety and effectiveness data on CIRT are derived from Japanese experience, where CIRT has been employed for unresected PCa since 1995 at the National Institute of Radiological Sciences (NIRS, Chiba, Japan), with excellent clinical toxicity and efficacy outcomes $(6,7)$.

Our study aimed to evaluate the impact of bladder and rectum filling variations in the CIRT dose-of-the-day

\footnotetext{
Abbreviations: $\mathrm{PCa}$, prostate cancer adenocarcinoma; OARs, organs at risk; CNAO, Centro Nazionale di Adroterapia Oncologica; IEO, Istituto Europeo di Oncologia; IRCCS, INT Fondazione IRCCS Istituto Nazionale dei Tumori; CIRT, carbon ion radiotherapy; IMRT, intensity modulated radiation therapy; RBE, radiobiological effectiveness; IGRT, image-guided radiation therapy; CT computed tomography; CBCT, cone beam computed tomography; FOV, field of view; sCT, synthetic computed tomography; pCT, planning computed tomography; CTV, clinical target volume; PTV planning target volume; MR, magnetic resonance; LEM, local effect model; DRR, digitally reconstructed radiograph; RO, radiation oncologist; $\mathrm{ROI}$, region of interest; $\mathrm{DVH}$, dose volume histogram; CV, coefficient of variation.
}

distribution of target and OARs in the context of our phase II mixed beam study for high-risk PCa. Additionally, our IGRT and patient preparation protocol reliability was assessed, evaluating the dose distribution reproducibility during the treatment course using CBCT data.

Dose evaluation on daily CBCTs for particle therapy is challenging due to increased scatter, beam hardening, Hounsfield unit (HU) inaccuracy and often small field-of-view (FOV) sizes (3). In this study, we proposed a method for dose-of-the-day calculation. Synthetic computed tomography (sCT) images were obtained by deforming pCT images into the daily CBCT frame of reference. Subsequently, the pCT Hounsfield units (HUs) were transferred to $\mathrm{sCT}$ to obtain the corresponding stopping power maps for CIRT dose calculation.

\section{MATERIAL AND METHODS}

\section{Patient Cohort}

We retrospectively analyzed treatment and imaging data of 16 consecutive patients, enrolled from 2016 to 2020 in a phase II study for CIRT boost treatment at CNAO, diagnosed with highrisk PCa according to the inclusion criteria previously described in Marvaso et al. (4). Patients' enrollment started after trial approval from all treating centers' Ethical Committees (8). The selected patients underwent carbon-ion boost at CNAO, followed by photon intensity-modulated radiotherapy (IMRT) at IEO or INT, and signed informed consents at the coordinating center prior to treatment. Daily imaging data sets of two patients were incomplete and excluded from the study.

\section{Target Definition and Treatment Planning}

The simulation CT acquired at CNAO was registered with the magnetic resonance (MR) image set for clinical target volume (CTV) delineation. The CTV included the prostate and the proximal third of the seminal vesicles. According to the protocol, planning target volume (PTV) was created by adding safety margins to the CTV, $5 \mathrm{~mm}$ in all directions. Rectum, bladder, bowel, and femoral heads were contoured as OARs for plan optimization with the following constraints for the boost phase: rectum $\mathrm{D}_{0.03 \mathrm{~cm} 3} \leq 100 \%, \mathrm{~V}_{16 \mathrm{~Gy}(\mathrm{RBE})} \leq 5 \%, \mathrm{~V}_{15 \mathrm{~Gy}(\mathrm{RBE})} \leq$ $20 \%$, bladder $\mathrm{D}_{0.03 \mathrm{~cm} 3} \leq 102 \%, \mathrm{~V}_{15 \mathrm{~Gy}(\mathrm{RBE})} \leq 35 \%$, femoral head $\mathrm{V}_{10 \mathrm{~Gy}(\mathrm{RBE})} \leq 15 \%$, and bowel $\mathrm{V}_{16.6 \mathrm{~Gy}(\mathrm{RBE})}=0 \%$. Target coverage objectives were PTV $\mathrm{D}_{98 \%} \geq 95 \%, \mathrm{D}_{0.03 \mathrm{~cm} 3} \leq 107 \%$, and median dose $\leq 102 \%$. Priority was given to OAR dose constraints over PTV coverage for boost plans. More details on the cumulative plan acceptance criteria can be found in Gugliandolo et al. (8).

A total dose of $16.6 \mathrm{~Gy}$ (RBE) in four fractions (4.15 Gy (RBE)/fraction, over 1 week) was delivered for the CIRT anticipated boost at CNAO. Afterward, patients received a whole-pelvis IMRT of $50 \mathrm{~Gy}$ in 25 fractions at IEO or INT. In this study, only the CIRT treatment phase was considered.

Since no gantry was available, two opposed lateral beams were delivered using a fixed horizontal line and rotating the couch. This beam orientation was chosen to avoid placing the rectum and bladder distally from the beam (9), where range uncertainties can strongly degrade the dose distribution (10). 
The planned dose was delivered with pencil beam scanning technique with lateral spot spacing and energy layer spacing of $2 \mathrm{~mm}$.

Treatment plans were optimized with RayStation v8.1 Treatment Planning System (TPS, RaySearch Laboratories Stockholm, Sweden). In order to mitigate range and setup uncertainties, a robust planning strategy was used based on minimax optimization, with setup and range uncertainties of 2 $\mathrm{mm}$ in all directions and 3\%, respectively (11). The RBEweighted dose was determined according to the local effect model LEM I (12) with an ideal $\alpha / \beta$ ratio of 2 Gy.

\section{Patient Positioning}

For each treated patient, a pCT was acquired on a SOMATOM Sensation Open CT scanner (Siemens Medical Systems, Germany) using a slice thickness of $2 \mathrm{~mm}$ with a pixel spacing of $0.98 \times 0.98 \mathrm{~mm}$ with machine parameters varying in the interval of $190-300 \mathrm{mAs}$ at $120-140 \mathrm{kV}$.

During the pCT acquisition and the whole treatment course, all patients were immobilized in supine position with a pelvic personalized solid thermoplastic mask (Renfu Medical Equipment, Guangzhou, China) fixed on an indexed base plate. In addition, customized large cushions (TOTIM ${ }^{\circledR}$ Patient Cushions Immobilization System, Essebi Medical SRL, Faetano, San Marino) were used in combination with knee and foot holders. An MR scan (Siemens Medical Systems, Germany) in the same setup condition was acquired after the pCT.

Patients were asked to empty the rectum with two micro enemas and drink $500 \mathrm{ml}$ of water after bladder voiding, 30 minutes before starting the CT examination and before each treatment fraction, in order to maintain consistency in rectum and bladder filling and have a comfortable position with the rigid mask.

Before treatment delivery, patient setup optimization was image-guided by acquiring double planar orthogonal $\mathrm{kV}$ images in the anteroposterior and right-left directions. The acquired images were aligned automatically (after a preliminary manual alignment when necessary) to the corresponding digitally reconstructed radiographs (DRRs). Subsequently, the six degrees of freedom robotic couch (13) compensated for the estimated translations and rotations. After patient setup adjustment (14) and before treatment delivery, a daily $\mathrm{CBCT}$ was acquired for soft tissue anatomical change inspection purposes. Each CBCT image was evaluated by a radiation oncologist $(\mathrm{RO})$, and if rectum or bladder filling was considered inadequate for treatment, the patient was asked to repeat the preparation.

CBCT images were acquired with a non-isocentric, customdesigned robotic imaging system (15). About 600 projective images were acquired during $220^{\circ}$ gantry rotation around the patient. CBCT acquisition parameters were set to $120 \mathrm{kVp}$ and $31 \mathrm{mAs}$. According to this clinical workflow, the bony anatomy imaged in the CBCTs is intrinsically co-registered to the pCT. The validity of this assumption was assessed by computing the $3 \mathrm{D}-3 \mathrm{D}$ registration between $\mathrm{CBCT}$ and $\mathrm{pCT}$, which resulted in sub-millimeter/degree of setup residuals.
CBCT volumetric images were reconstructed with a spatial resolution of $1 \times 1 \times 1 \mathrm{~mm}$ and stored in Meta Image file format (text-based tagged file format ".mha"). For this study, the axial field of view (FOV) of the CBCT was reduced to a diameter of $200 \mathrm{~mm}$ in order to mitigate the truncation artifacts. In addition, CBCTs were converted in DICOM format with the same frame of reference of the planning CT, for loading into the RayStation TPS.

\section{Synthetic CT of the Day and Dose Recalculation}

The sCTs were created by deforming the initial pCT (target image set) on each daily CBCT (reference image set) by using the ANAtomically CONstrained Deformation Algorithm (ANACONDA) implemented in RayStation TPS (16). We mainly exploited the structure-based approach of the algorithm discarding the image intensity information during algorithm computation and focusing the deformation on the CBCT FOV. At first, femur heads and other pelvic bones, including sacrum and coccyx, contoured as landmarks on the pCT, were cropped according to the CBCT FOV. Subsequently, these contours were rigidly transferred on each CBCT. CTV, PTV, rectum, bladder, and bowel were manually re-contoured on each $\mathrm{CBCT}$ for each patient by an in-training RO and verified by an experienced RO. PTV, rectum, bladder, and the FOV-cropped bony structures were used as controlling regions of interest (ROIs) to drive the deformation. The deformation vector field was estimated with a resolution comparable to pCT of $1 \times 2 \times 1 \mathrm{~mm} /$ voxel in rightleft, inferior-superior, and posterior-anterior directions, respectively. Each resulting deformation vector field was exported from RayStation and applied to the pCT using Plastimatch (version 1.9.0), yielding a sCT. The resulting sCT mimicked the original pCT outside the CBCT FOV, where the deformation vector field was zero, while inside the FOV the pCT was deformed according to the soft tissue geometry as detected in the CBCT. The planned dose distribution was recalculated on each sCT to have a reasonable estimation of the patient delivered dose on the anatomy of each treatment session. The resulting sCTs were evaluated in terms of quality of the deformation, analyzing the correspondence of bladder, rectum, prostate, and bones position between sCTs and CBCTs and confirming the absence of the deformation field outside the CBCT FOV. A schematic representation of the method described here to generate a sCT for dose-of-the-day recalculation is depicted in Figure 1.

\section{Data Analysis}

Inter-fractional changes in patient anatomy were estimated computing bladder, rectum, and PTV volume variations between planning and treatment fractions. The coefficient of variation $(\mathrm{CV})$ of the structure volumes was investigated for the whole patient cohort and each patient separately.

Average DVHs were obtained for the PTV, rectum, and bladder for pCT and sCT dose distributions, and the standard deviation of the population was computed at each dose level. 


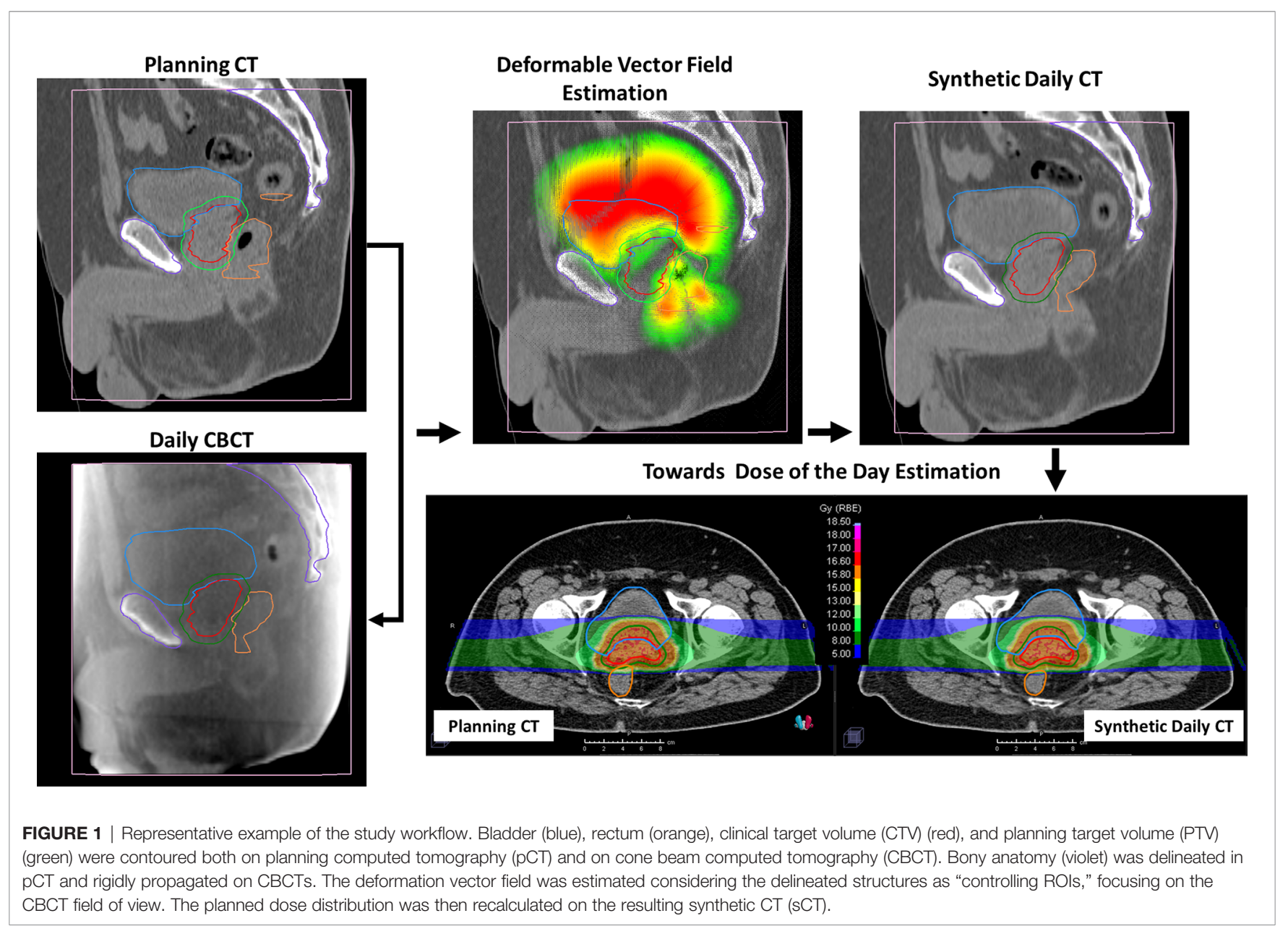

Treatment plan dose constraints for the bladder and rectum, together with PTV coverage objectives, were verified on each recalculation plan. The following DVH-based metrics were extracted for the pCT and each sCT: $V_{5 G y(R B E)}, V_{10 G y(R B E)}$, $\mathrm{V}_{15 \mathrm{~Gy}(\mathrm{RBE})}$, and $\mathrm{V}_{16 \mathrm{~Gy}(\mathrm{RBE})}$ for rectum and bladder, and $\mathrm{D}_{95 \%}$, $\mathrm{D}_{98 \%} \mathrm{D}_{50 \%}, \mathrm{D}_{2 \%}$, and $\mathrm{D}_{0.03 \mathrm{~cm} 3}$ for PTV. sCT metric deviations from the planning values were evaluated with the Wilcoxon signed-rank test.

Finally, correlations between the bladder and rectum volumes, and the corresponding DVH metrics, were evaluated with the Spearman correlation test.

\section{RESULTS}

A total of 56 CBCTs were evaluated: 4 daily CBCTs for each of the 14 enrolled patients.

Volumes of bladder and rectum varied across patients and fractions. Figure 2A shows the bladder, rectum, and PTV simulation contours on the planning $\mathrm{CT}$, with the contours derived from the daily CBCTs superimposed, for $\mathrm{P} 7$, as an example. For bladder, a higher-volume variability across patients and treatment fractions was found (ranging from 44 to $455.5 \mathrm{~cm}^{3}, \mathrm{CV}: 54.2 \%$ ), as compared to rectum (ranging from 22.9 to $65.3 \mathrm{~cm}^{3}, \mathrm{CV}: 25.4 \%$ ) and PTV (ranging from 60.2 to $146.5 \mathrm{~cm}^{3}, \mathrm{CV}: 21.0 \%$ ). Figure 2B shows the distribution of organ volumes at $\mathrm{pCT}$ and during the treatment course, considering the whole patient cohort. According to the Wilcoxon test, there was no significant difference in the bladder, rectum, and PTV median volumes between the pCT and the CBCTs acquired during the treatment course (Table 1).

Considering each patient separately, the volumes of bladder and rectum on the CBCTs were different compared to the volume on the $\mathrm{pCT}$. Volume variations during the CIRT course were mostly patient-dependent: $\mathrm{CV}$ of bladder volume ranged from $8 \%$ for P13 to $70 \%$ for P6. Similarly, CV for rectum varied between 2\% for patient P11 to 35\% for P6 (Figure 3).

In addition, no significant difference was found when comparing planned and treatment average DVHs as in Figure 4, and median DVH metrics between $\mathrm{pCT}$ and CBCTs for rectum, bladder, and PTV (Table 1).

Distributions of DVH parameters for bladder, rectum, and PTV are presented separately for each patient in Figures 5-7, respectively. pCT treatment plans always satisfied all OAR constraints. Bladder $\mathrm{V}_{15 \mathrm{~Gy}(\mathrm{RBE})} \leq 35 \%$ was always met except for P11 for three out of four fractions. Rectum $V_{16 \mathrm{~Gy}(\mathrm{RBE})} \leq 5 \%$ 
A

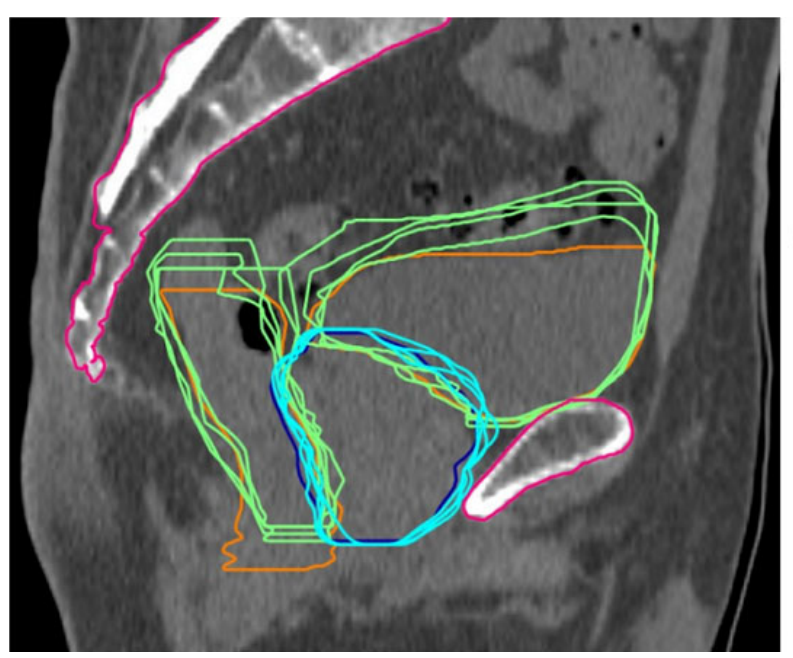

B

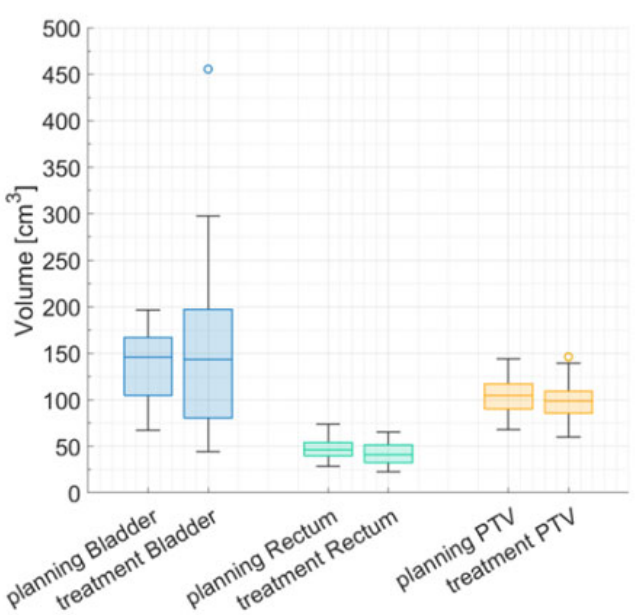

FIGURE 2 | (A) Representative planning image with planning computed tomography (CT) contours of planning target volume (PTV) (blue line) and rectum and bladder (orange lines), with contours delineated on cone beam CTs (CBCTs) superimposed (light-blue and green lines). (B) Boxplots for bladder, rectum, and PTV volume at planning CT and CBCT for the whole cohort.

TABLE 1 I Comparison of volumes and dose-volume histogram (DVH) indices of bladder, rectum, and planning target volume (PTV) at planning CT (pCT) and at synthetic CTs (sCTs) for all the patients.

\begin{tabular}{|c|c|c|c|c|c|c|c|}
\hline & & \multicolumn{3}{|c|}{ Bladder } & \multicolumn{3}{|c|}{ Rectum } \\
\hline & & $\begin{array}{c}\text { Planning } \\
\text { median (IQR) }\end{array}$ & $\begin{array}{c}\text { Treatment } \\
\text { median (IQR) }\end{array}$ & $\begin{array}{c}\text { Wilcoxon test } \\
\text { p-value }\end{array}$ & $\begin{array}{c}\text { Planning } \\
\text { median (IQR) }\end{array}$ & $\begin{array}{c}\text { Treatment } \\
\text { median (IQR) }\end{array}$ & $\begin{array}{c}\text { Wilcoxon test } \\
\text { p-value }\end{array}$ \\
\hline Volume & $\mathrm{cm}^{(3)}$ apex & $146.3(62.3)$ & 143.5 (116.6) & 0.924 & $46.5(14.6)$ & $40.8(18.8)$ & 0.125 \\
\hline \multirow[t]{6}{*}{ DVH metrics } & V16 Gy(RBE) [\%] & $14.1(6.5)$ & $12.6(10.0)$ & 0.659 & $4(1.1)$ & $2.8(2.8)$ & 0.165 \\
\hline & V15 Gy(RBE) [\%] & $17.5(9.0$ & $16.1(11.8)$ & 0.654 & $11.9(7.4)$ & $9.8(9.1)$ & 0.336 \\
\hline & V10 Gy(RBE) [\%] & 26.8 (10.9) & $27.1(16.0$ & 0.724 & 28.7 (11.5) & $26.5(14.2)$ & 0.463 \\
\hline & V5 Gy(RBE) [\%] & $35.2(13.8)$ & $33.9(20.8)$ & 0.774 & $36.2(11.4)$ & $35.2(19.1)$ & 0.592 \\
\hline & & \multicolumn{3}{|c|}{ PTV } & & & \\
\hline & & $\begin{array}{l}\text { Planning } \\
\text { median (IQR) }\end{array}$ & $\begin{array}{l}\text { Treatment } \\
\text { median (IQR) }\end{array}$ & $\begin{array}{c}\text { Wilcoxon test } \\
\text { p-value }\end{array}$ & & & \\
\hline Volume & $\mathrm{cm}^{(3)}$ apex & $104.8(26.7)$ & $98.5(23.9)$ & 0.45 & & & \\
\hline \multirow[t]{4}{*}{ DVH metrics } & D98\% [\%] & $96.5(1.9)$ & $95.6(3.0)$ & 0.148 & & & \\
\hline & D95\% [\%] & $98.6(0.9)$ & $98.2(2.4)$ & 0.354 & & & \\
\hline & D50\% [\%] & $99.8(0.3)$ & $99.8(0.3)$ & 0.595 & & & \\
\hline & D2\% [\%] & $100.6(0.3)$ & $100.6(0.3)$ & 0.699 & & & \\
\hline
\end{tabular}

and $\mathrm{V}_{15 \mathrm{~Gy}(\mathrm{RBE})} \leq 20 \%$ dose constraints were met in $47(84 \%)$ and $50(80 \%)$ recalculation plans, respectively. In only one case (P3), rectum dose constraints were not met in all the recalculation plans. When considering high doses, no hot spots were found either in the rectum or in the bladder, with $\mathrm{D}_{0.03 \mathrm{~cm} 3}$ below $100 \%$ and $102 \%$ of the prescribed dose, respectively.

Median PTV $\mathrm{D}_{98 \%}$ and $\mathrm{D}_{95 \%}$ for treatment planning were not significantly higher compared to the sCT-recalculated plans (Table 1). The PTV coverage objective $\left(D_{98 \%} \geq 95 \%\right)$ was achieved in 12 of the 14 pCT optimized plans (85.7\%), while this dose criterion was met in 34 of $56(60.7 \%)$ cases in the recalculation plans (Figure 7). Concerning the hot spots, $\mathrm{D}_{0.03 \mathrm{~cm} 3}$ was lower than $107 \%$ of the prescription dose in all the cases.

In order to assess how bladder and rectum filling might affect OAR dose distribution, we considered these OARs' overlap volumes with the 16-Gy (RBE) and 15-Gy (RBE) isodoses for each patient treatment plan. No correlation was found between the variation in rectum and bladder volume as compared to the $\mathrm{pCT}$ and the variation in the OAR overlap volume. In particular, rectum correlation coefficients were 0.19 ( $p$-value: 0.156$)$ and $0.34(p$ value: 0.011), respectively, whereas for bladder, correlation coefficients were 0.10 (p-value: 0.444$)$ and 0.11 (p-value: 0.425$)$. 

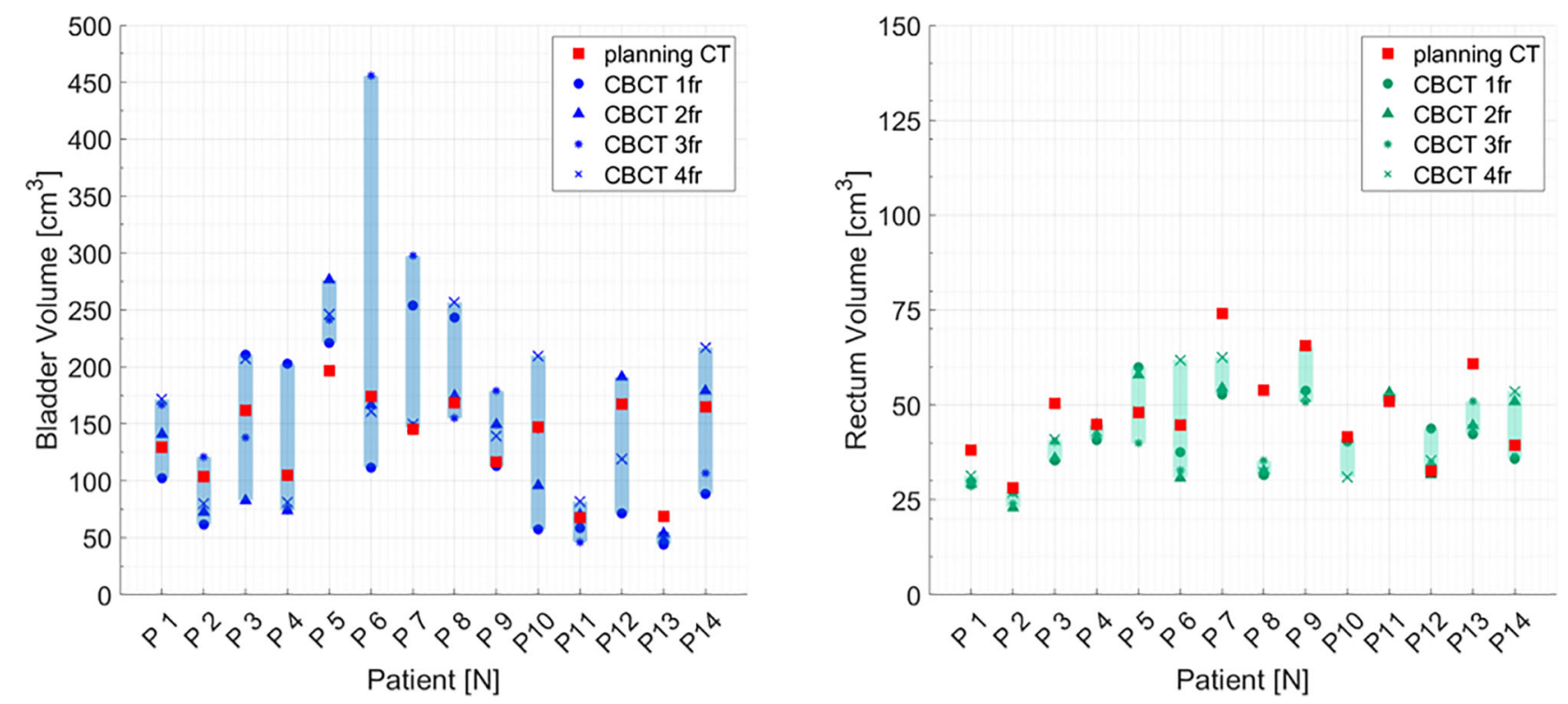

FIGURE 3 | Bladder and rectum volume at planning CT (red squares) and at each cone beam CT (dark symbols) for all the patients separately. Graphical shades indicate the extent of volume variability.

On the contrary, the correlation between the absolute volumes of rectum and bladder with the respective absolute volumes receiving $16 \mathrm{~Gy}(\mathrm{RBE}), 15 \mathrm{~Gy}(\mathrm{RBE}), 10 \mathrm{~Gy}(\mathrm{RBE})$, and 5 Gy (RBE) increased as the considered dose decreases. For rectum, in particular, the correlation coefficient increased from 0.40 to 0.55 ( $p$-value $<<0.001$ ), while for bladder the coefficient increased from 0.34 ( $p$-value: 0.0038 ) to 0.61 ( $p$-value $<<0.001)$. The greater the OAR absolute volume, the greater the absolute volume receiving low doses.

A statistically significant correlation was found between the bladder absolute volume variation at $\mathrm{pCT}$ and $\mathrm{CBCT}$ and the DVH metric variation at $\mathrm{pCT}$ and CBCTs expressed as a percentage of the corresponding volumes when considering the entire patient cohort. If bladder volume decreased during treatment, the percentage of bladder volume receiving $16 \mathrm{~Gy}$ (RBE), 15 Gy (RBE), 10 Gy (RBE), and 5 Gy (RBE) increased (correlation coefficient $>0.5$, p-value $<<0.001$ ).

\section{DISCUSSION}

The impact of inter-fractional variation in urinary bladder volume and rectum filling on daily dose distribution during CIRT for high-risk pCA was investigated in 14 patients enrolled in the AIRC IG 14300 grant frame (4).

For dose calculation on daily CBCT, we generate a sCT to overcome various CBCT limitations that forbid CIRT dose calculation. So far, sCT has been reported for proton dose calculations (17). This approach, based on Deformable Image Registration (DIR), has the advantage of not introducing $\mathrm{HU}$ inaccuracies in sCT images since the deformation does not modify the original pCT numbers. The distribution of $\mathrm{HU}$ between pCT and sCT was consistent for each contoured structure (bladder, rectum, PTV). Nonetheless, we are aware that this method has several issues that have to be addressed. Firstly, the air pockets eventually found in the $\mathrm{CBCT}$ images were propagated in the sCT, or conversely, when air pockets were found in the $\mathrm{pCT}$ they were not propagated in the SCT (18). However, considering the beam irradiation geometry, such areas were not included in the beam path. Secondly, the limited CBCT FOV resulted in incomplete patient external contour. Assuming that simulation CT was a reasonable estimation of the patient anatomy not included in the CBCT, pCT data were used to compensate for this missing information (19). Moreover, DIR could deform bones when large deformation occurs close to bony structures (20). To overcome any unrealistic bone deformations, we included bone ROI as a shape constraint during DIR computation.

One of the main goals of this study was to evaluate the validity of our IGRT approach consisting in bone-matching followed by CBCT acquisition. At present, the primary IGRT approach for moving targets, including pCA treatment positioning verification in particle therapy, consists in DRR bone-matching or target (prostate)-matching using implanted fiducials. Using orthogonal $\mathrm{X}$-ray images for patient positioning verification is the standard procedure for CIRT in most centers (21). In our investigation, we found only very slight displacements of the PTV center of mass as determined by the contours on the $\mathrm{SCT}$ obtained from CBCT, with median (IQR) of $0.1(0.3) \mathrm{mm},-0.3(1.3) \mathrm{mm}$, and $-0.3(0.5)$ $\mathrm{mm}$ toward the right, anterior, and inferior directions, respectively. These values were smaller than the prostate displacement found with IGRT techniques for photon IMRT prostate treatment (22). One possible explanation could be patient mask immobilization for CIRT, which might strongly reduce bowel and pelvic anatomy changes and thus prostate 


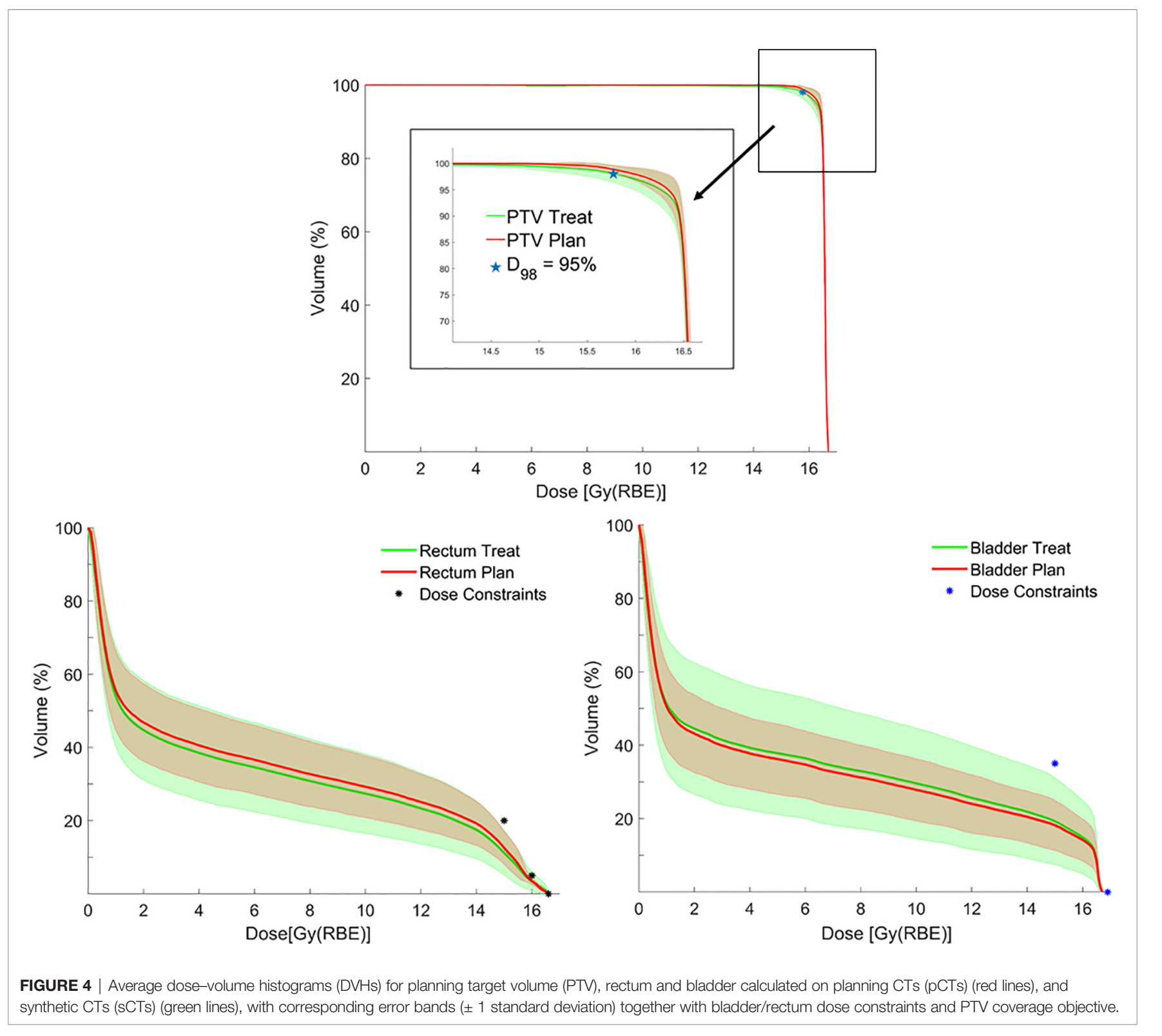

displacement (2). Looking at our results in terms of target displacement, our PTV margins (5 $\mathrm{mm}$ in all directions) appear suitable for the considered patient population.

Maeda et al. (23) reported that prostate-matching was more reliable than the bone-matching approach regarding rectum dose constraint adherence and target coverage in prostate proton therapy delivered with geometry of two opposed beams. At CNAO, thin golden filaments called Gold Anchor ${ }^{\mathrm{TM}}$ were investigated to assess the improvement in prostate position verification (24). Their visibility on both $\mathrm{CT}$ and radiographic images and the possible perturbation of the carbon ion beams were investigated through tests in an anthropomorphic phantom and turned out to be acceptable. In the future, we aim to implant the Gold Anchor ${ }^{\mathrm{TM}}$ seeds in some patients to perform further validation of our IGRT protocol, comparing prostate-matching versus bone-matching approach for patient positioning before CBCT acquisition.
Another essential purpose of the presented study was the validation of our patient preparation protocol. Bladder filling or rectal gas movement may influence the prostate position and therefore affect the target coverage. In parallel, the rectum and bladder could eventually receive unwanted hot spots if such OARs move in the high-dose treatment area resulting from the two-lateral opposed beam irradiation geometry.

At present, optimal rectum and bladder filling conditions for prostate external beam photon RT are still debated. In our study, patient preparation aimed at achieving a comfortable bladder filling compatible with pelvic mask compression and treatment duration while preventing major rectum and bladder volume variation compared to simulation conditions. Despite that precise fluid intake instructions were given to the patients, bladder volume varied considerably during the CIRT course in the analyzed patient cohort-as for P7, a patient with important 

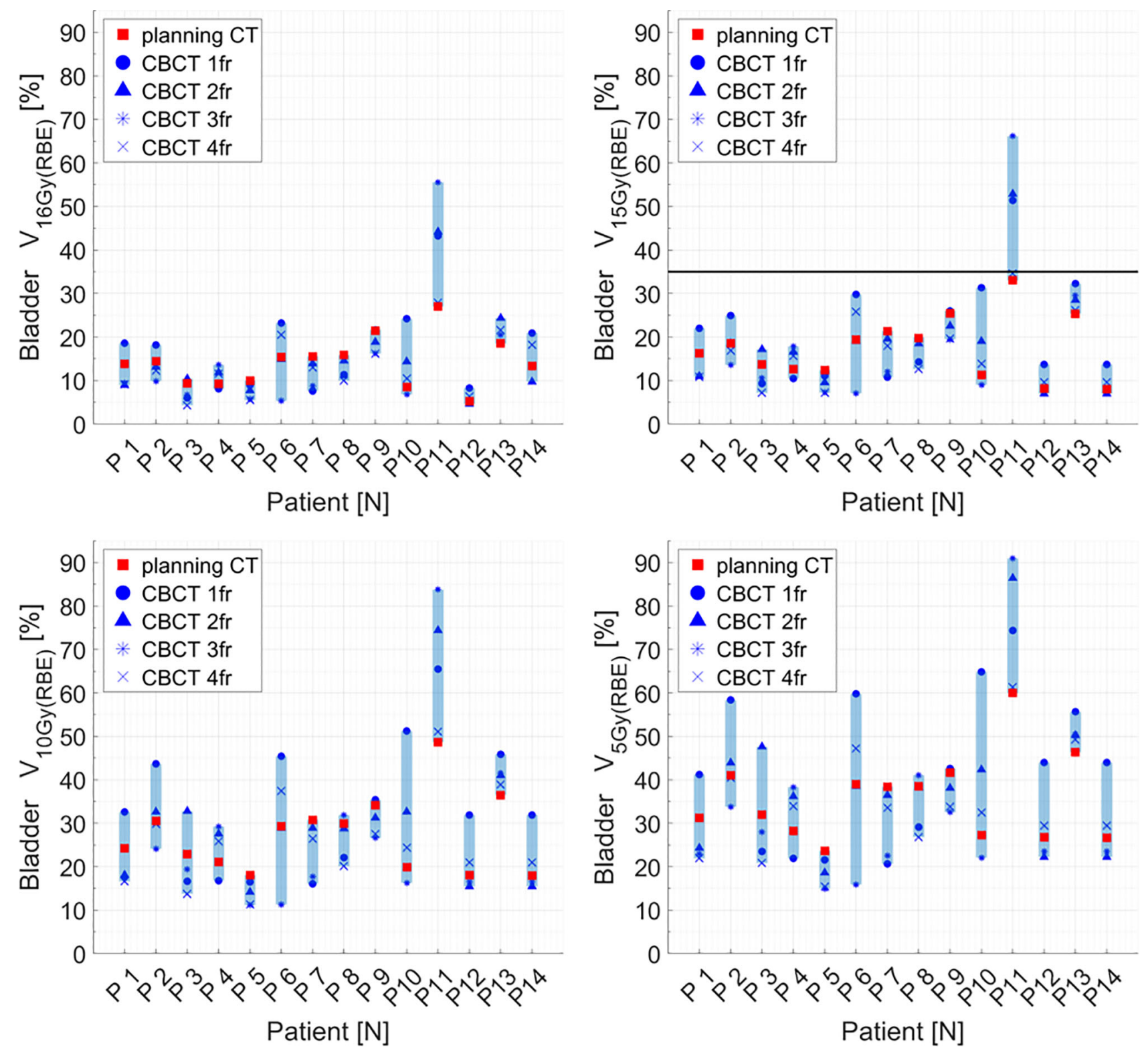

FIGURE 5 | Distribution of bladder dose-volume histogram (DVH) metrics ( $\left.V_{16 G y(R B E)}, V_{15 G y(R B E)}, V_{10 G y(R B E)}, V_{5 G y(R B E)}\right)$ for each patient at planning CT (red squares) and at each synthetic CT (sCT) (dark markers). Colored range bars indicate the maximum variation extent within a single patient. The bladder constraint $\mathrm{V}_{15 \mathrm{~Gy}(\mathrm{RB} E)}$ $\leq 35 \%$ is indicated as a black line. CBCT, cone beam computed tomography; fr, fraction.

obstructive urine retention. To a lesser extent, also the volume of the rectum varied among the fractions during the CIRT course. However, no statistically significant increase of the dose to OARs at treatment was observed.

Similarly, no major dose degradation in terms of PTV dose was observed. Even if the PTV D98\% $\geq 95 \%$ goal failed in $40.7 \%$ of the recalculated plans, only slight deviations were found in D95\% for the pCT optimized plans, with a mean relative difference of $0.1 \%$, considering all the treatment fractions in the series of patients.

At CNAO, patients with high-risk localized prostate cancer are currently being treated either with a photon-CIRT mixed beam approach or with a full course of CIRT of 66.4 Gy (RBE) delivered in 16 fractions (4 days/week) to the prostate and seminal vesicles (25), according to Japanese experience (26, 27). Our findings showed that the setup and IGRT protocols described here appeared to be suitable also for patients treated with a full course of CIRT.

In our investigation, we focused exclusively on the residual inter-fraction anatomical variations after bony alignment and did not consider intra-fraction motion. However, several recent studies on cine-MR imaging extensively reported that prostate intrafraction motion could affect the target dose distribution $(28,29)$. 

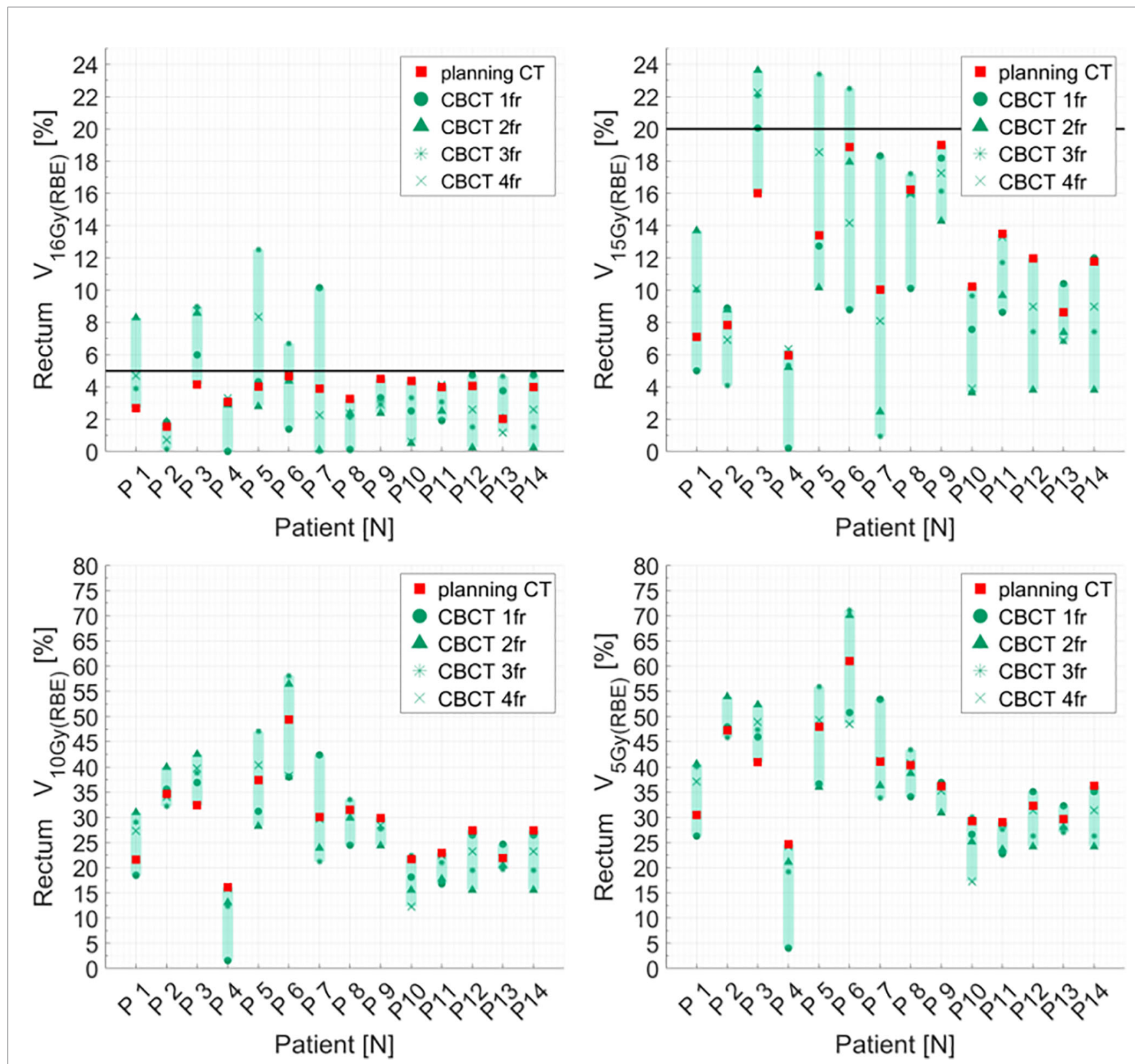

FIGURE 6 | Distribution of rectum dose-volume histogram (DVH) metrics $\left(V_{16 G y(R B E)}, V_{15 G y(R B E)}, V_{10 G y(R B E)}, V_{5 G Y(R B E)}\right)$ for each patient at planning CT (red squares) and at each synthetic CT (sCTs) (dark markers). Colored range bars indicate the maximum variation extent within a single patient. The rectum constraints $V_{16 G y(R B E)}$ $\leq 5 \% V_{15 G y(R B E)} \leq 20 \%$ are indicated as a black line. CBCT, cone beam computed tomography; fr, fraction.

These studies concluded that 5-mm PTV margins were adequate to guarantee target coverage even during long-lasting treatments $(>10 \mathrm{~min})$. Since the presented CIRT prostate treatment time was approximately 2 min per beam and a rigid thermoplastic mask was used for patient immobilization, we do not expect the intra-fraction prostate motion to impact the dose distribution significantly.

We are aware that one of the limitations of the current analysis is the small patient cohort. However, the enrolment of patients in the phase II protocol described here is ongoing, and we aim to validate the current data in a larger patient series as soon as more patients are treated.

\section{CONCLUSION}

The dosimetric impact of anatomical changes on CIRT was assessed in the context of our phase II mixed beam study for high-risk pCa patients. Dose deviations as determined by bladder and rectum filling variations demonstrated that the preparation 

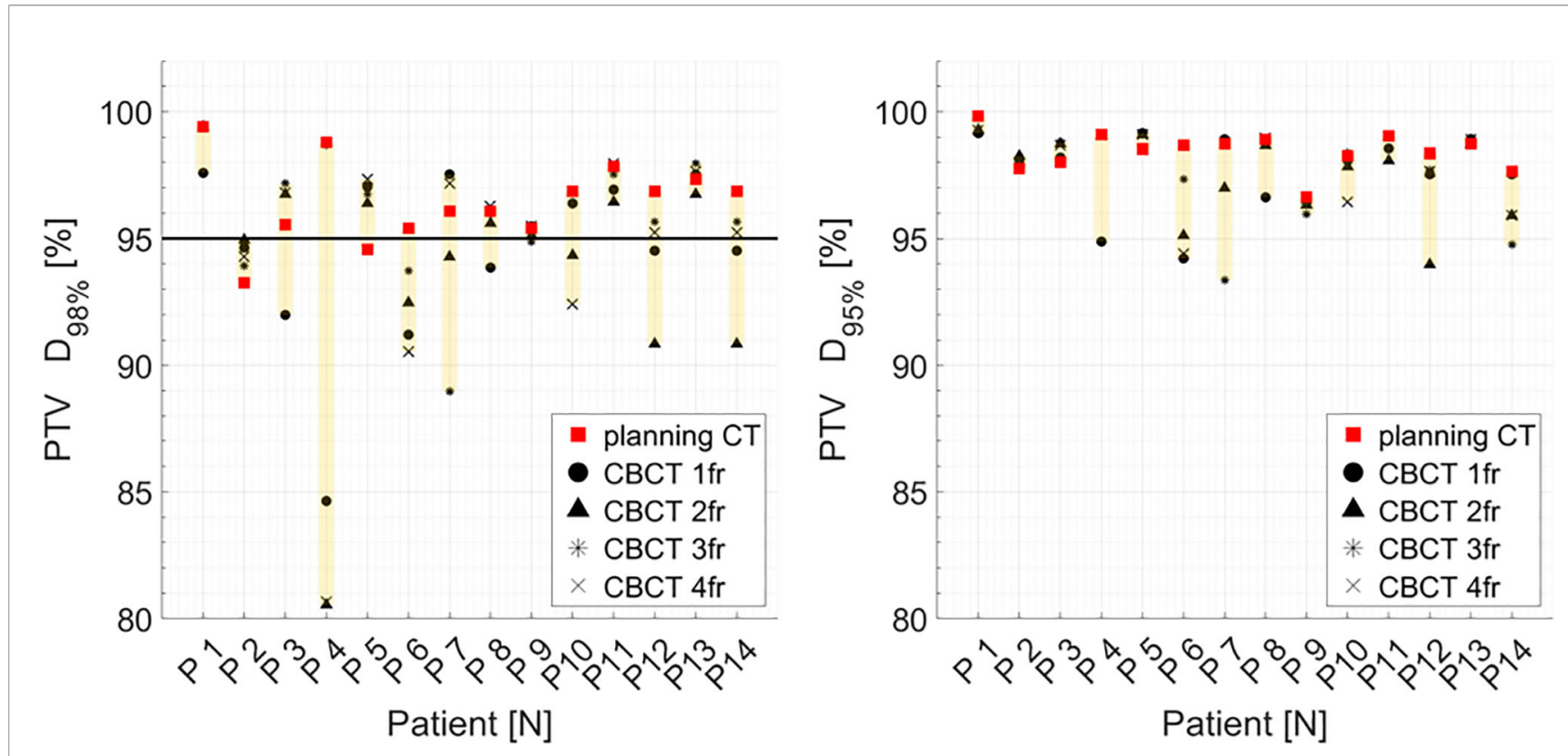

FIGURE 7 | Distribution of PTV DVH metrics ( $\mathrm{D}_{98 \%}, \mathrm{D}_{95 \%}$ ) for each patient at planning CT (red squares) and at each synthetic CT (sCTs) (dark markers). Colored range bars indicate the maximum variation extent within a single patient. Target coverage objective PTV D98\% $\geq 95 \%$ is indicated as a black line. CBCT, cone beam computed tomography; fr, fraction.

protocol and the IGRT approach described here could generate reproducible dose distributions in terms of target coverage and OARs sparing.

The generation of sCTs from daily CBCTs for dose-of-the-day calculation in CIRT for high-risk pCA is clinically feasible. The proposed method is suitable for an adaptive treatment strategy providing a daily treatment plan based on the actual anatomy.

\section{DATA AVAILABILITY STATEMENT}

The raw data supporting the conclusions of this article will be made available by the authors, without undue reservation.

\section{ETHICS STATEMENT}

The studies involving human participants were reviewed and approved by Comitato Etico Pavia, Fondazione IRCCS Policlinico San Matteo, Pavia, Italy, notification no. 20140025096. The patients/participants provided their written informed consent to participate in this study.

\section{REFERENCES}

1. Ten Haken RK, Forman JD, Heimburger DK, Gerhardsson A, McShan DL, Perez-Tamayo C, et al. Treatment Planning Issues Related to Prostate Movement in Response to Differential Filling of the Rectum and Bladder. Int J Radiat Oncol Biol Phys (1991) 20(6):1317-24. doi: 10.1016/0360-3016(91)90244-x

2. Yokoyama A, Kubota Y, Kawamura H, Miyasaka Y, Kubo N, Sato H, et al. Impact of Inter-Fractional Anatomical Changes on Dose Distributions in Passive

\section{AUTHOR CONTRIBUTIONS}

Conceptualization, SR, RR, and BV. Methodology, SR, RR BV, SM, and AP. Software, SR and RR. Validation, SR, RR, BV, SM, AP, FP, and MC. Formal analysis, SR, RR, SM, BV, AP, GB, and MC. Data curation, SR, RR, BV, SM, and AP. Investigation, SR, RR, SM, FP, AB, EM, AP, CP, GM, MP, MZ, SC, BA, TG, EP, RV, $\mathrm{GB}, \mathrm{FC}, \mathrm{MC}, \mathrm{BAJF}, \mathrm{EO}$, and RO. Writing-original draft preparation, SR, RR, and BV. Writing-review and editing, SR, RR, SM, FP, AB, EM, AP, CP, GM, MP, MZ, SC, BA, TG, EP, RV, $\mathrm{GB}, \mathrm{FC}, \mathrm{MC}, \mathrm{BJ}, \mathrm{EO}$, and RO. All authors contributed to the article and approved the submitted version.

\section{FUNDING}

This study was partially supported by a research grant from Associazione Italiana per la Ricerca sul Cancro (AIRC IG 14300): "Carbon ion boost followed by pelvic photon radiotherapy for high risk prostate cancer", registered at ClinicalTrials.gov (NCT02672449).

Carbon-Ion Radiotherapy for Prostate Cancer: Comparison of Vertical and Horizontal Fields. Front Oncol (2020) 10:1264. doi: 10.3389/fonc.2020.01264

3. Giacometti V, Hounsell AR, McGarry CK. A Review of Dose Calculation Approaches With Cone Beam CT in Photon and Proton Therapy. Phys Med (2020) 76:243-76. doi: 10.1016/j.ejmp.2020.06.017

4. Marvaso G, Jereczek-Fossa BA, Vischioni B, Ciardo D, Giandini T, Hasegawa A, et al. Phase II Multi-Institutional Clinical Trial on a New Mixed Beam RT Scheme of IMRT on Pelvis Combined With a Carbon Ion Boost for 
High-Risk Prostate Cancer Patients. Tumori (2017) 103(3):314-8. doi: $10.5301 /$ tj.5000587

5. Nomiya T, Tsuji H, Kawamura H, Ohno T, Toyama S, Shioyama Y, et al. A Multi-Institutional Analysis of Prospective Studies of Carbon Ion Radiotherapy for Prostate Cancer: A Report From the Japan Carbon Ion Radiation Oncology Study Group (J-Cros). Radiother Oncol (2016) 121:28893. doi: 10.1016/j.radonc.2016.10.009

6. Akakura K, Tsujii H, Morita S, Tsuji H, Yagishita T, Isaka S, et al. Phase I/II Clinical Trials Of Carbon Ion Therapy for Prostate Cancer. Prostate (2004) 58 (3):252-8. doi: 10.1002/pros.10328

7. Ishikawa H, Tsuji H, Kamada T, Yanagi T, Mizoe JE, Kanai T, et al. Working Group for Genitourinary Tumors. Carbon Ion Radiation Therapy for Prostate Cancer: Results of a Prospective Phase II Study. Radiother Oncol (2006) 81 (1):57-64. doi: 10.1016/j.radonc.2006.08.015

8. Gugliandolo SG, Marvaso G, Comi S, Pepa M, Romanò C, Zerini D, et al. Mixed-Beam Approach for High-Risk Prostate Cancer: Carbon-Ion Boost Followed by Photon Intensity-Modulated Radiotherapy. Dosimetric and Geometric Evaluations (AIRC IG-14300). Phys Med (2020) 76:327-36. doi: 10.1016/j.ejmp.2020.07.012

9. Schwarz M, Molinelli S. What can Particle Therapy Add to the Treatment of Prostate Cancer? Phys Med (2016) 32(3):485-91. doi: 10.1016/j.ejmp. 2016.03.017

10. Sakai M, Kubota Y, Saitoh JI, Irie D, Shirai K, Okada R, et al. Robustness of Patient Positioning for Interfractional Error in Carbon Ion Radiotherapy for Stage I Lung Cancer: Bone Matching Versus Tumor Matching. Radiother Oncol (2018) 129(1):95-100. doi: 10.1016/j.radonc.2017.10.003

11. Fredriksson A, Forsgren A, Hårdemark B. Minimax Optimization for Handling Range and Setup Uncertainties in Proton Therapy. Med Phys (2011) 38(3):1672-84. doi: 10.1118/1.3556559

12. Krämer M, Scholz M. Treatment Planning for Heavy-Ion Radiotherapy: Calculation and Optimization of Biologically Effective Dose. Phys Med Biol (2000) 45(11):3319-30. doi: 10.1088/0031-9155/45/11/314

13. Pella A, Riboldi M, Tagaste B, Bianculli D, Desplanques M, Fontana G, et al. Commissioning and Quality Assurance of an Integrated System for Patient Positioning and Setup Verification in Particle Therapy. Technol Cancer Res Treat (2014) 13(4):303-14. doi: 10.7785/tcrt.2012.500386

14. Ricotti R, Pella A, Tagaste B, Elisei G, Fontana G, Bonora M, et al. Long-Time Clinical Experience in Patient Setup for Several Particle Therapy Clinical Indications: Management of Patient Positioning and Evaluation of Setup Reproducibility and Stability. Br J Radiol (2020) 93(1107):20190595. doi: 10.1259/bjr.20190595

15. Fattori G, Riboldi M, Pella A, Peroni M, Cerveri P, Desplanques M, et al. Image Guided Particle Therapy in CNAO Room 2: Implementation and Clinical Validation. Phys Med (2015) 31(1):9-15. doi: 10.1016/j.ejmp. 2014.10.075

16. Kadoya N, Nakajima Y, Saito M, Miyabe Y, Kurooka M, Kito S, et al. MultiInstitutional Validation Study of Commercially Available Deformable Image Registration Software for Thoracic Images. Int J Radiat Oncol Biol Phys (2016) 96(2):422-31. doi: 10.1016/j.ijrobp.2016.05.012

17. Landry G, Nijhuis R, Dedes G, Handrack J, Thieke C, Janssens G, et al. Investigating CT to CBCT Image Registration for Head and Neck Proton Therapy as a Tool for Daily Dose Recalculation. Med Phys (2015) 42(3):135466. doi: $10.1118 / 1.4908223$

18. Ziegler M, Nakamura M, Hirashima H, Ashida R, Yoshimura M, Bert C, et al. Accumulation of the Delivered Treatment Dose in Volumetric Modulated Arc Therapy With Breath-Hold for Pancreatic Cancer Patients Based on Daily Cone Beam Computed Tomography Images With Limited Field-of-View. Med Phys (2019) 46(7):2969-77. doi: 10.1002/mp.13566

19. de Smet M, Schuring D, Nijsten S, Verhaegen F. Accuracy of Dose Calculations on kV Cone Beam CT Images of Lung Cancer Patients. Med Phys (2016) 43(11):5934. doi: 10.1118/1.4964455
20. Motegi K, Tachibana H, Motegi A, Hotta K, Baba H, Akimoto T. Usefulness of Hybrid Deformable Image Registration Algorithms in Prostate Radiation Therapy. J Appl Clin Med Phys (2019) 20(1):229-36. doi: 10.1002/acm2.12515

21. Li Y, Kubota Y, Tashiro M, Ohno T. Value of Three-Dimensional Imaging Systems for Image-Guided Carbon Ion Radiotherapy. Cancers (Basel) (2019) 11(3):297. doi: 10.3390/cancers 11030297

22. Li M, Ballhausen H, Hegemann NS, Ganswindt U, Manapov F, Tritschler S, et al. A Comparative Assessment of Prostate Positioning Guided by ThreeDimensional Ultrasound and Cone Beam CT. Radiat Oncol (2015) 10:82. doi: 10.1186/s13014-015-0380-1

23. Maeda Y, Sato Y, Minami H, Yasukawa Y, Yamamoto K, Tamamura H, et al. Positioning Accuracy and Daily Dose Assessment for Prostate Cancer Treatment Using in-Room CT Image Guidance at a Proton Therapy Facility. Med Phys (2018) 45(5):1832-43. doi: 10.1002/mp.12858

24. Panizza D, Ciocca M, Mirandola A, Molinelli S, Mairani A, Gallio E, et al. P087: Are Fiducial Markers for IGRT Suitable for Use in Particle Beam Radiotherapy? In: Proceedings to the 53rd Annual Meeting for the Particle Therapy Cooperative Group (PTCOG) 8-14 June 2014: Hosted by the Shanghai Proton and Heavy Ion Center and the Fudan University Shanghai Cancer Center. Int J Part Ther (2014) 1(2):408-670. doi: 10.14338/IJPT.14-PTCOG.1

25. Choi K, Molinelli S, Russo S, Mirandola A, Fiore MR, Vischioni B, et al. Rectum Dose Constraints for Carbon Ion Therapy: Relative Biological Effectiveness Model Dependence in Relation to Clinical Outcomes. Cancers (Basel) (2019) 12(1):46. doi: 10.3390/cancers12010046

26. Okada T, Tsuji H, Kamada T, Akakura K, Suzuki H, Shimazaki J, et al. Carbon Ion Radiotherapy in Advanced Hypofractionated Regimens for Prostate Cancer: From 20 to 16 Fractions. Int J Radiat Oncol Biol Phys (2012) 84 (4):968-72. doi: 10.1016/j.jijrobp.2012.01.072

27. Nomiya T, Tsuji H, Maruyama K, Toyama S, Suzuki H, Akakura K, et al. Phase I/II Trial of Definitive Carbon Ion Radiotherapy for Prostate Cancer: Evaluation of Shortening of Treatment Period to 3 Weeks. Br J Cancer (2014) 110(10):2389-95. doi: 10.1038/bjc.2014.191

28. de Muinck Keizer DM, Kerkmeijer LGW, Willigenburg T, van Lier ALHMW, Hartogh MDD, van der Voort van Zyp JRN, et al. Prostate Intrafraction Motion During the Preparation and Delivery of MR-Guided Radiotherapy Sessions on a 1.5T MR-Linac. Radiother Oncol (2020) 151:88-94. doi: 10.1016/j.radonc.2020.06.044

29. De Muinck Keizer DM, Willigenburg T, der Voort van Zyp JRN, Raaymakers BW, Lagendijk JJW, Boer JCJ. Seminal Vesicle Intrafraction Motion During the Delivery of Radiotherapy Sessions on a 1.5 T MR-Linac. Radiother Oncol (2021) 162:162-9. doi: 10.1016/j.radonc.2021.07.014

Conflict of Interest: The authors declare that the research was conducted in the absence of any commercial or financial relationships that could be construed as a potential conflict of interest.

Publisher's Note: All claims expressed in this article are solely those of the authors and do not necessarily represent those of their affiliated organizations, or those of the publisher, the editors and the reviewers. Any product that may be evaluated in this article, or claim that may be made by its manufacturer, is not guaranteed or endorsed by the publisher.

Copyright (๐ 2021 Russo, Ricotti, Molinelli, Patti, Barcellini, Mastella, Pella, Paganelli, Marvaso, Pepa, Comi, Zaffaroni, Avuzzi, Giandini, Pignoli, Valdagni, Baroni, Cattani, Ciocca, Jereczek-Fossa, Orlandi, Orecchia and Vischioni. This is an openaccess article distributed under the terms of the Creative Commons Attribution License (CC BY). The use, distribution or reproduction in other forums is permitted, provided the original author(s) and the copyright owner(s) are credited and that the original publication in this journal is cited, in accordance with accepted academic practice. No use, distribution or reproduction is permitted which does not comply with these terms. 